\title{
Sport Performance as a Domain of Creative Problem Solving for Self- Organizing Performer-Environment Systems
}

\author{
Robert Hristovski*,a $^{\text {,a }}$ Keith Davids ${ }^{\mathrm{b}}$, Pedro Passos $^{\mathrm{c}}$ and Duarte Araújo \\ ${ }^{a}$ Faculty of Physical Culture, University of St. Cyril and Methodius, Macedonia \\ ${ }^{b}$ School of Human Movement Studies, Queensland University of Technology, Australia \\ ${ }^{c}$ Faculty of Human Kinetics, Technical University of Lisbon, Portugal
}

\begin{abstract}
In this paper we discuss the role of nonlinear pedagogy in explaining and facilitating creative behaviors within the sport performance context. Some research results are also highlighted as examples of creative problem solving within sports such as martial arts and rugby union. Within the framework of nonlinear pedagogy, creative behaviors may occur as a consequence of a specific constellation of interacting constraints that impinge on the performer-environment system. The relaxation of constraints enhances the potential for exploratory behavior and enables greater fluency and flexibility, increasing the probability of discovering atypical functional solutions to a task goal. In the example of team games, we highlight that critical values of interpersonal distance in attacker-defender dyads define a region that affords high metastability and reorganization of dyad actions. Here idiosyncratic performance solutions are created by immediate constraints of the system. Also, the performance context offered by the team is of utmost importance for creating possibilities for action at an individual level. In general we emphasize that analysis of the performer-environment system can provide a full account of the emergence of creative behaviors in sport performance contexts.
\end{abstract}

Keywords: Creativity in sport, nonlinear pedagogy, complex dynamic systems, performer-environment system, constraints, affordance, exploratory behavior.

\section{INTRODUCTION}

A reasonable answer to the question, "What is the similarity between team and individual goal-directed actions in sport?" may be that both, i.e. teams and individuals, are systems which face ill-defined problems. There is more than one functional solution to the task goal for each system. Whether the goal is a team scoring a point or a single movement organization to kick or catch a ball there are usually many ways to complete these tasks. This makes evolution of sports actions ill-defined and such problems lack single definite functional solutions.

Guilford, 1956 [1] conceptualized creativity as the ability to generate different responses to a task as a consequence of divergent thinking, rather than finding a single predetermined solution, which requires convergent thinking. Sports performance problems invariably belong to the former class of concepts, emphasizing creativity. Because of the complexity of environmental, personal and performance goal interactions, there is always more than one possible effective solution to the problem at hand and the effectiveness (i.e., goodness of fit) of solutions are not pre-determined. Moreover, athletes often intentionally act in such a way to change the task constraints, i.e. opponent's behaviours, to create a situation that affords efficient action corresponding to some subgoal or the main goal of the game [2]. In this way, athletes

*Address correspondence to this author at the Faculty of Physical Culture, University of St. Cyril and Methodius, Macedonia; Tel: 38923113654; Fax: 38923117538; E-mail: robert_hristovski@yahoo.com are not in fixed interactions with their environment within which a single correct solution is predetermined, but on the contrary, a performance goal can usually be achieved by multiple solutions.

Torrance [3] emphasized three key properties of creative thought/behavior: fluency, flexibility and originality. Fluency is defined as a generation of a large number of alternate solutions to a problem; flexibility is generation of a variety of classes of solutions; and originality refers to the atypicality of solutions to the problem. Atypicality may be defined with respect to one's own dynamic action landscape or to the socio-cultural landscape in a performance domain. One such dynamical landscape is represented on Figs. (2B and 3). In other words, a performer manifests one type of atypicality when discovering an action that was not a part of her/his previous performer-environment action landscape, but was extant in the wider socio-cultural context, e.g. a child discovers a back-heel pass in soccer. The second type exists when a performer discovers a solution that is also new in the wider socio-cultural context, e.g. a "Fosbury Flopp" technique in high jumping [4]. Currently, the majority of researchers concur that a creative product or behavior is co-characterized by two key dimensions: novelty (i.e. originality) and functionality (i.e. usefulness, effectiveness, appropriateness, success, or adequacy) [5-11]. Although Torrance's practical tests of 'thinking creatively in action and movement' [3] have proven to be adequate for assessment of motor creativity, especially in children, they alone are not able to explain how a creative behavior emerges under task constraints. 
The multiplicity of task solutions in sport can be interpreted within the framework of complex dynamical systems (CDS). CDS requires the time-space problem to be typically rich in solutions and single solutions are characterized as specific cases emerging under suitable conditions. This property of complex adaptive systems in which the goal can be attained by different means and structural components is referred to as degeneracy [12]. This contrasts with theories which are forced to make ad hoc adjustments with every single issue that has to be explained. For example, in some theories of decision making or action selection $[13,14]$ the multiple solutions (or options) have to be, independently of the theory, postulated and put forth ad hoc, on the basis of the common human experience. Once the number and types of solutions are fixed in an ad hoc manner, the system has to choose among them, without the possibility to find a novel solution.

Another characteristic of the CDS approach is the focus on discovering general principles and not general trait structures or mechanisms of systems under scrutiny. This is a requirement of a general theory which in conjunction with constraints, i.e. boundary conditions, explains the idiosyncratic dynamics which lead to creative behaviors. The idiosyncrasy of individual creative behavior has been already acknowledged even within the traditional line of research on this topic [9]. The key characteristic of CDS approach may show pertinent in solving problems that face the research in many domains of creativity different than the sports realm. For example, the multistability which is a direct consequence of nonlinear interactions between the system components is a natural explanation of the potential for the exploratory fluency and flexibility of solutions in any domain of creativity. The subjectivity of the creative process follows from the idiosyncratic interaction of personal, environmental and task constraints of each individual-environment interaction. The moment of insight is a classical example of a state transition when a qualitative reorganization and quick emergence of novel information within the system takes place $[15,16]$. The self-assembling hierarchy is already present in the very notion of the collective variable which governs the elementary system components, but is reciprocally formed by their interactions. Such context dependant hierarchy has a much bigger potential to explain the emergence of new ideas and concepts, than traditional rigid hierarchies of universal encapsulated modules within the brain. Investigating the time scales relations of different processes (i.e. quick vs. slow degrees of freedom) involved in creative behavior [9] can result in fundamental results in a similar vein as it happened in studies of learning and development [17]. Moreover, while traditional approach conceives created constructs as mere combinatorial inventions [18] the notion of the collective variable naturally explains the Gestalt, i.e. the wholistic, character of the invented product. These are some characteristics which CDS theory offers to the creativity research in general.

One theoretical development within the sports sciences, underpinned by CDS principles and formalisms, is the constraints-led perspective on learning [19-24]. More specifically, the ecological dynamics of decision making, action and learning $[25,26]$ finds its practical embodiment in a nonlinear pedagogy [22,27]. Within the framework of nonlinear pedagogy, a skill and more generally talent, is not a trait possessed by individuals alone but a property of the athlete-environment system subject to changing constraints [28-30]. In the context of creativity, defined generally as the discovery of novel actions in athletes and teams, this type of emergent behavior has been demonstrated in previous research [31-35]. In such a way creativity, may be envisioned as the highest emergent type of adaptive behavior of an athlete-environment system.

\section{ECOLOGICAL DYNAMICS AND INNOVATION IN MULTILEVEL DYNAMICS OF AFFORDANCES}

In ecological psychology, information is perceived as the link between opportunities to act (i.e., affordances) and action capabilities (i.e., effectivities), capturing the fit between performer constraints and invariant properties of the environment [36]. Affordances are specific to the individual at the ecological scale of analysis [25,37-39]. So, perceiving an affordance is to perceive how one can act when faced with a particular set of performance conditions in the environment. Therefore, creative movement behaviours are predicated, not only on the ability to detect relevant sources of information that offers individuals an opportunity to act, but also to act in order to keep a desired response within a range of action possibilities [38]. This understanding of human behaviour expresses the mutual coupling between perception and action sub-systems. Performers prospectively control their behaviors by producing movements that are guided on the basis of information about future states of affairs $[36,40,41]$.

Fruitful insights from dynamical systems theory have been merged with ideas of ecological psychology to enhance understanding of the emergence of movement behaviours in sports $[23,25,42,43]$. For example, in team sports, ecological dynamics seeks to understand how functional patterns of coordinated behavior emerge through a process of selforganization in attacker and defender interactions under specific task and environmental constraints [25,42]. In the study of creative movement this approach advances the understanding by examining how physical variables may constrain neurobiological systems (e.g., performers) and social neurobiological systems (e.g., teams) to change between different stable states of organization. Nonlinear complex systems such as athlete-environment systems exhibit properties called multistability and metastability. These properties enable many coexisting behavioral solutions for the same configuration of constraints and some of these co-existing performance solutions may contain different levels of novelty.

When a performer perceives information that specifies an affordance for action (such as in team games when an attacker attempts to move behind a marking defender with the ball), the performer-environment (dyadic) system relaxes to one specific stable attractor, as it concomitantly ignores the remaining co-existing states (other more or less novel action possibilities for dyadic system organisation) [25,28]. In sport, metastable dynamics of system organization emerge from the inter-twined processes of action, cognition, and perception. Due to continuous changes in interacting performance constraints, the stability of a system attractor can be changed so that system organization may become unstable and allow the emergence of novel stable states (defenders 
can rearrange to re-stabilise dyadic systems quickly) [44]. The emergence of novelty sometimes may be expressed by a critical point at which one action is no longer more stable than another, forcing behaviour to become unstable or change [28,31]. Changes in constraints can lead a system towards bifurcation points where choices emerge as more specific information becomes available, constraining the environment-athlete system to switch to a different path of behaviour [25]. Emergent transitions in system organization towards greater novelty reflect the psychological process of creativity in sport [25]. That is, phase transitions in the course of human behaviour can be understood as affordance boundaries, i.e. areas of emerging and dissolving action opportunities.

\section{CREATIVITY LEVELS AND EXPERTISE RELAT- EDNESS}

The traditional Big $\mathrm{C}$ to little c creativity dichotomy, where Big C stands for "extreme forms of originality" and little c for "everyday creativity has been criticized over the years. Recently, Beghetto and Kaufmann [45], proposed a 4level structure of creative achievement, i.e. mini c creativity, little $\mathrm{c}$ creativity, Pro $\mathrm{C}$ creativity and Big $\mathrm{C}$ creativity. Within this framework, mini c creativity is inherent to the learning process and is usually manifested in learners when they discover a performance solution with respect to their actual intrinsic dynamics [46]. This can be captured by the nonlinear pedagogy framework which puts special emphasis on the key role of the interaction of the personal constraints with task and environmental constraints in learning process. Mini c creativity in sports may be defined as a discovery or adaptation of known techniques to one's own personal constraints. In this way the learning process is not a passive copying process but active exploratory development where the general pattern of a known technique is being assimilated into a specific athlete-environment configuration of constraints rendering an idiosyncratic style.

Little c-creativity on the other hand refers to the creative behavior which one can observe in athlete-environment interactions of non-expert athletes, e.g. the domain of recreational sport, where problem solving space is ill-defined and multiple functional solutions exist. Yet, as this athleteenvironment context is not at an expert level, i.e. emerging solutions are not of an expert type, these solutions differ in some aspects to expert Pro $\mathrm{C}$ creative behaviors. This behavior is defined by emerging patterns which can be found in athlete-environment contexts where the fit of athlete to environmental demands is represented by highly skilled, flexible and integrated emerging actions. Big $\mathrm{C}$ creative events are possibly the easiest to define and recognize in sports. The invention of a novel and functional technique which relatively quickly diffuses in the domain is a good example of this kind of creativity. The emergence of new track and field techniques and gymnastic movement forms are examples of of Big C creativity. Truly Big C creative behavior does come from self-channeling of the performer, often going counter to influences by the socio-cultural ambience (teacher or coach). This is understandable because if the behavior is a part of the extant socio-cultural ambience it cannot be highly original. This means that Big $\mathrm{C}$ behaviors are underpinned by statisti- cally rare confluence of personal, task and environmental constraints which elicit the invention.

On the other hand, techniques which can be explicitly imposed on the athlete may form part of a socio-cultural task-solution landscape of an athlete-environment system (for example, a national style of performing). Because of its channelling role, the socio-cultural landscape, however, may limit the set of solutions within which athletes may choose to explore. Therefore, socio-cultural task-solution landscapes can be used to assess the degree of originality of the potential novel solution as well as to analyse their role as social constraints on athletes [35].

It is interesting to note that the path from mini-ccreativity to higher forms of creativity is not predetermined and not a continuous passing from mini $\mathrm{c}$ to Pro $\mathrm{C}$ or Big $\mathrm{C}$ type. Neither of these types of creativity occurs in timely, ordered phases.

As in other fields of performance, in sport there are cases where Big C creative behaviors are not constrained by the level of expertise. This caveat highlights the importance of a 'hands-off' approach to coaching [24], especially in early phases of sports training and motor learning. Indeed, cases like the invention of Fosbury Flop and O'Brian techniques in track and field show that in many but not all cases a high level of expertise is neither a necessary, nor sufficient condition for establishing highly novel athlete-environment relationships, although successive mastering of an invented movement form is needed for high performance outcomes. Fosbury started to develop the Flop after 5-6 years of training of the scissors technique with a short period of trying the straddle technique. In his own words Dick Fosbury, "was really lousy with that (straddle) style"(4). In other words the interaction of his personal constraints with the task constraints of the straddle technique were such as to produce low performance outcomes. This case shows how a ratelimiting constraint may be lifted to trigger exploratory behaviors and, as a result, the invention of a highly novel solution such as the Flop technique. Hence, Fosbury's practice efforts were oriented toward a straddle technique when he transited to the already obsolete scissors technique whose gradual modification led him to the Flop technique (4). Parry O'Brian was developing his expertise in football, for nearly the same amount of time, i.e 5-6 years, and attended shotput events only in high school tournaments before he started to explore other task constraint sets (e.g. initial position of the body and arm) and eventually invented the O'Brien Glide shot put technique. These are potent examples which show how expertise level and creative behavior are not necessarily related [47], and how the facilitation of early forms of mini and little c- creativity are sometimes crucial for Big C creative behavior to emerge.

On the other hand creative behaviors in the form of situational functional adaptations of actions in team sports almost invariably are connected to a high level of expertise. For example, the breadth of attention is a requisite ability of top soccer players for their creative task solutions [48]. However, if elementary soccer skills are not stabilized, the breadth of attention would be suppressed by immediate need of ball control, which on the other hand would severely diminish the capacity of highly adaptive tactical behavior. 
These observations suggest that manifestations of sports creativity are multifaceted and need a thorough and systematic investigation.

\section{HOW NOVELTY EMERGES WITHIN COMPLEX DYNAMICAL SYSTEMS: THE ROLE OF EXPLORA- TORY BEHAVIOR.}

The system's capacity for exploration satisfies the minimal criterion of creativity suggesting that a performance solution (e.g. target movement or technique) or exploration, (e.g. a list of different movement patterns), should not be explicitly imposed on the performer by an external agent. Exploratory behavior can be readily observed in the most elementary motor performances of athletes. For example, such behavior is present in reconfigurations of goal-directed activities of performers such as quasi-isometric exercises under increasing fatigue constraints [49]. Athletes under such constraints continuously search for novel performer environment configurations with a goal to delay the exercise termination point. Although this kind of exploration activity seems a far cry from truly creative acts in sports, such as the invention of a new vault, it is necessary to recognize that the exploratory process is fundamentally the same.

Three elementary properties of CSD lead to exploratory behaviour for novel stable solutions. The first is nonlinearity of component interactions. For novel states or forms of behavior to occur, the system's components have to be nonlinearly coupled to enable multistability. This stems from the fact that only nonlinearly coupled systems form multiple stable states resilient to small perturbations.

Second, a large number of constraints configurations are also present within and out of the system which form the control space of the system. In human performance contexts such as sport, particularly influential are the task constraints (e.g. the goal, performer-environment contact surfaces, equipment), personal (e.g. body morphology, relative strength, motives, emotions) and environmental constraints such as gravity, temperature, social ambience [27]. As the interaction of the task, personal and environmental constraints is different for each individual the landscape and the exploratory behavior of each athlete-environment system will be different. Within some intervals of constraint values the systems perform stably in one or several modes. The most probable values of such modes are called attractors (stable states of organization). However, as the constraints reach some configuration of values, the system will be destabilized and it cannot perform stably in the previous regime but spontaneously transits to another behavioral solution/s, i.e. attractors. In other words, behavioral solutions that were previously highly probable may become extinct and new modes of behavior may emerge. This is a point of bifurcation, a critical point, or more generally a critical manifold.

Third property is the intrinsic variability of CDS. As in all real complex systems there is noise, arising from processes that exist on shorter time scales than the observed behavior, there is always a possibility of switching behavior into another mode as a result. The larger the magnitude of background noise, the greater the probability of switching, and the shorter the time of the system passing through, i.e. exploring, all available behavioral modes. Note that in this case the noise itself is sufficient to enable the system to visit the multiple stable solutions, instead of doing it through the change of constraints. Generally the intrinsic variability of the CSD and the manipulation of constraints, i.e. the control space, may be used to enhance the exploratory breadth of CSD. This would enable larger flexibility, fluency and possibly originality (atypicality) to emerge within the system.

\section{DISCOVERY OF ACTION SOLUTIONS. A CASE OF MINI C CREATIVITY IN SPORTS.}

Creative action fluency and flexibility were demonstrated in a heavy-bag punching task in boxing $[35,43]$. Performers were unfamiliar with a heavy-bag punching task and were instructed to apply any punch on the heavy-bag that was afforded them. They were asked to carry out 60 punches at each of 10 different distances from the heavy-bag. What was revealed in the study was that different punching actions were discovered and ceased to exist for various performer target scaled distances.

A heavy-bag punching task has a stable goal of directing the actions toward a specified target area and enabling a fisttarget impact under afforded angles. These angles were defined as collective variables, i.e. order parameters, of the performer-environment system. In Hristovski et al. [31] it was revealed that actions emerged and dissolved at the bifurcation points determined by the scaled performer - target distance: $\mathrm{D}=$ performer's arm length / physical distance to target. The continuous punching from each distance took around 40 seconds. From a dynamical system's perspective it could be construed that the dynamical goal-directed state of performers was stable for about 40 seconds, which defined the observation time per distance. Various transient single and double hand combinatorial patterns in the form of multiplets (i.e. more than three punches one after another), triplets and doublets developed on time scales of a few seconds to a few hundredths of milliseconds, respectively, while single actions occurred in the range of 70 to 110 milliseconds. Time scale is a time distance between two events measured in decades, e.g. $0.07-0.7,0.7-7$, or 7-70 seconds). In other words, heavy-bag punching was a dynamical system state spanning several time scales, i.e. decades, with one stable task goal state encompassing all other metastable states living on shorter time scales. That is to say, while the general goal of the task was stable on the observation time scale of tens of seconds, it was satisfied by different transient actions emerging on shorter time scales. This property showed how under body-scaled constraints of performers, a flexible solution manifold was self-assembled. This solution dynamical structure was assembled by nested correlated performerheavy-bag action patterns (see Fig. 1). The value of overlap of the general task goal level $\mathrm{q}_{0}=0.45$ showed a weak constraint on the particular actions. This means that the task goal $\mathrm{q}_{0}$ enabled and was satisfied by a wide variety of actions. The lower levels showed increasingly higher values, meaning that they were more stringently spatially constrained by increasingly specifying informational and anatomical constraints. Interestingly, such correlation structure is characteristic of soft-assembled dynamics of complex brain networks [50], characterized by globally weak integration and increasingly stronger localized coupling [35]. 

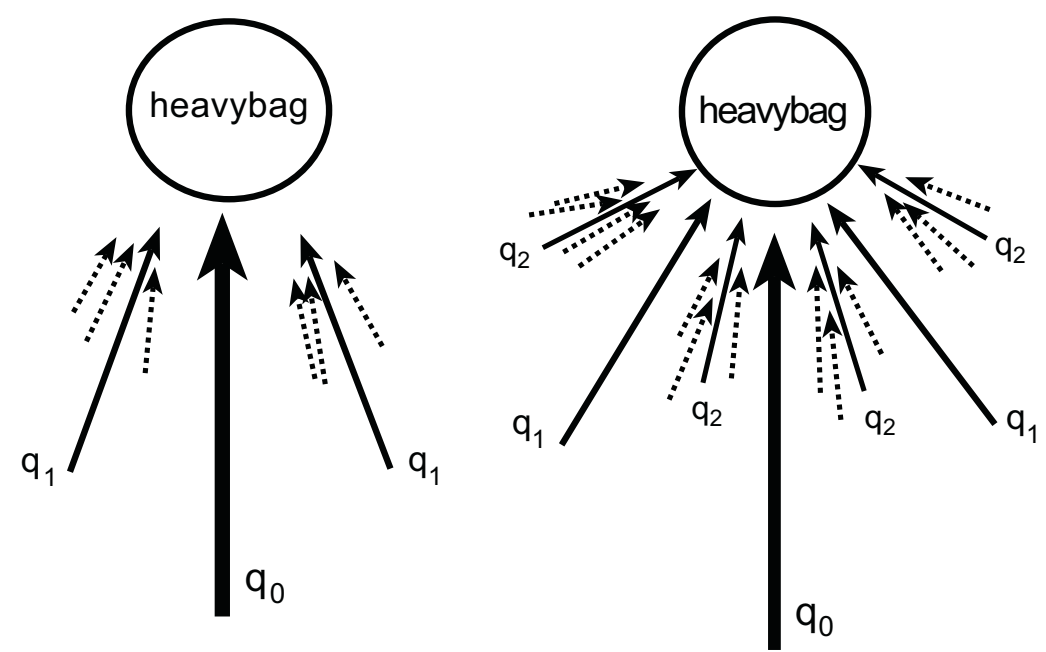

Fig. (1). A. Nested correlated action configurations for $D=1.12$. B the same for $D=0.86$. A. $q_{0}=$ level of task goal; $q_{1}=$ level of left-right jabs; dashed lines = level of variations of left-right jabs. B. $\mathrm{q}_{0}$ the same as in A. $\mathrm{q}_{1}$ level of left-right arm actions; $\mathrm{q}_{2}$ level of left-right hooks and jabs; and dashed lines = level of variations of hooks and jabs. X and Y are real space coordinates. See also Figs. (2 and 3 ).

It is clear that the two key aspects of creative behavior by Torrance [3], i.e. fluency and flexibility are strongly dependent on the active constraints impinging on the performer environment system. While for $\mathrm{D}=1.12$ (see Fig. 1A) only two actions of the same class were discovered (left and right jabs), for a value of $\mathrm{D}=0.86$ more $(\mathrm{n}=4)$ actions were discovered and used from different action categories, i.e. jabs and hooks (see Fig. 1B). This observation signifies that fluency as well as flexibility were changed by the suitable set of constraints. At $\mathrm{D}=0.6$ these properties of the exploring performer - environment system were maximized because, in addition to the 4 actions observed at $\mathrm{D}=0.86$, the left and right uppercuts also emerged, i.e. a new class of actions was discovered by performers [31,35]. One can see in Fig. (1A) and $\mathrm{B}$ that directions/orientations of a performer's punches with the heavy-bag formed nested correlated clusters. These correlated actions may be envisioned similar to a hierarchical principal component analysis fashion. In hierarchical PCA a number of variables due to mutual correlations form variables of higher order and hence reduce the number of the degrees of freedom of the system. Correlations of orientations of punches may be defined as a collective variable which relates the angles of front fist-heavybag collisions performed sequentially or simultaneously. This collective variable $\mathbf{q}_{\mathrm{ii}}$, in other words, is defined as the overlap between actions $\mathbf{i}$ and $\mathbf{j}$ or classes of actions $\mathbf{i}$ and $\mathbf{j}$ and may be defined in a multidimensional case as a cosine of the angle between two random vectors [35].

In our simple case, taking into account only the fist-target orientation angle, what was revealed after 60 punches, is an existence of a continual dynamical hierarchical state of collective variables (see Fig. 2), with those on the higher level constraining those on the lower level. In dynamical systems theory such higher order variables reciprocally constrain the behavior of the lower level variables [51]. The dashed ar-



Fig. (2). A. Collective variables hierarchy for $\mathrm{D}=0.86$ revealed by average linkage algorithm. $\mathbf{B}$. The exploratory landscape of the discovered actions hierarchy. (with kind permission from Nonlinear Dynamics, Psychology and Life Sciences). 
rows represent individual punches and they are correlated so to form classes of actions like jabs and hooks ( $\mathrm{q}_{2}$ level). This level further correlates to form general left or right arm punches $\left(\mathrm{q}_{1}\right.$ level) and further these levels correlate to form the $\mathrm{q}_{0}$ level of general goal directed punching activity.

Note, however, that the dynamic hierarchy has a different structure for different task constraints, i.e. performer-target scaled distances (Fig. 1A and B). This fact demonstrates that the hierarchy of nested dynamic states are constraintsdependent and that the discovery of novel actions may be facilitated by their manipulation. In other words we can see how a nested intentional activity has been soft-assembled, i.e. emergent, as a result of a specific configuration of constraints. Novel intentional actions with this framework are dynamical products of the performer-environment interactions.

\section{EXPLORATORY BREADTH OF PERFORMER- ENVIRONMENT SYSTEM}

The exploratory breadth of the athlete - environment system may be measured as a diffusion strength $\mathrm{Q}$. The diffusion strength is defined as being equal to the average escape probability over all possible action solutions $Q=\left\langle W_{\mathrm{e}}\right\rangle$. Escape probabilities for each solution are defined as $W_{\mathrm{e}}=1$ $W_{\mathrm{c}}$, where $W_{\mathrm{c}}$ is the conditional probability of staying inside the same attractor [35]. In other words, $W_{\mathrm{c}}$ measures the trapping strength of the action solution, i.e. the probability of sequentially performing the same action. The larger the average escape probability $\left\langle W_{\mathrm{e}}\right\rangle$, the larger the exploratory breadth $Q$ of the system. Alternatively, when stationary conditional distribution is not known,e.g., when state probabilities are still not stationary, one may use another measure based on the average overlap $\mathbf{q}$ of action solutions, i.e. $\mathbf{Q}=$ $1 /<q_{i j}>$ defined for some time interval. The subsequent actions within the performer - heavybag system may be seen as a exploratory dynamics hopping between attractors on different hierarchical levels, or as a random walk on a tree along its branches (see Fig. 3).

From Fig. (3) it can be seen that if hopping is conducted within some of attractors on the $\mathrm{q}_{3}$ level, that means that the system explores one mode of actions, here for example, hooks. In other words it explores only the subtle different configurations of one class of actions. If the system has a hopping dynamics that includes the $\mathrm{q}_{2}$ level than exploration encompasses couplings between different classes of actions, say hooks and jabs, or all three classes including uppercuts. Thus, the value of overlap q defines the fundamental level of the exploratory dynamics. Depending on the set of constraints the whole set of explored actions, as we noted before, is different. That is to say the exploratory landscape and the dynamics within are very sensitive to the manipulation of the key constraints. It is the interacting constraints that shape the exploratory dynamics of the performer - environment system.

\section{DISCOVERY OF A NOVEL PUNCH IN THE MAR- TIAL ARTS DOMAIN UNDER RELAXED CON- STRAINTS: A CASE STUDY OF INNOVATION.}

Within the framework of ecological dynamics of creative behavior invention or innovation may be defined as an action or movement that, until it has been performed, was not a part of the performer's intrinsic dynamic landscape or the sociocultural landscape [35]. In an experiment conducted more recently we applied the same paradigm as used in Hristovski et al. [31]. Four participants, unfamiliar with the heavybag punching task, were asked to perform 60 punches at a static heavybag from 10 different distances. For the last 4 scaled distances, i.e. $0.0 \leq \mathrm{D} \leq 0.4$, the experiment was repeated, however, the heavybag was stochastically moved in perpendicular direction to the central visual line of the performer by an assistant. The maximum elongation of the heavy-bag crossed the shoulder width of the performer (see Fig. 4).

In this study, 4 performers discovered all actions that belong to the extant action landscape of boxing, akin to the Hristovski et al. study [43]. Note that these actions belonged to the socio-cultural task-solution landscape traditionally classified as boxing actions. However, two performers under the relaxed task constraints of the stochastic motion of the heavy-bag, and without any specific verbal instructions, discovered a novel punching movement, which is considered illegal and hence atypical, within boxing sport, but is similar to the back-fist strike in karate, the so called uraken-uchi punch (see Fig. 4). They were able to suppress the habitual

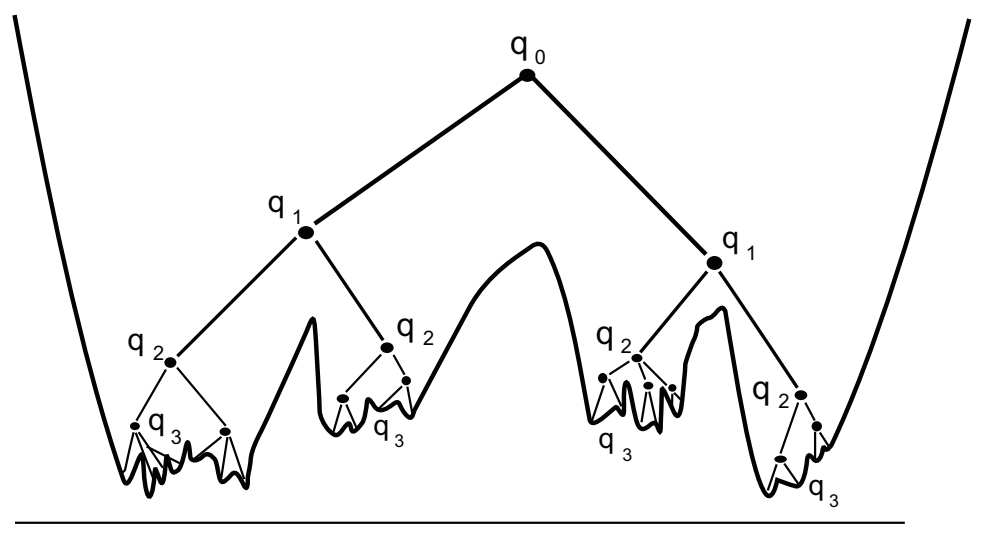

Fist - Heavybag Configurations

Fig. (3). A one-dimensional schematic representation of the performer - heavybag hierarchical action landscape. The exploratory dynamics may be defined as a hopping dynamics in the landscape or as a random walk on a tree. (with kind permission from Nonlinear Dynamics, Psychology and Life Sciences). 


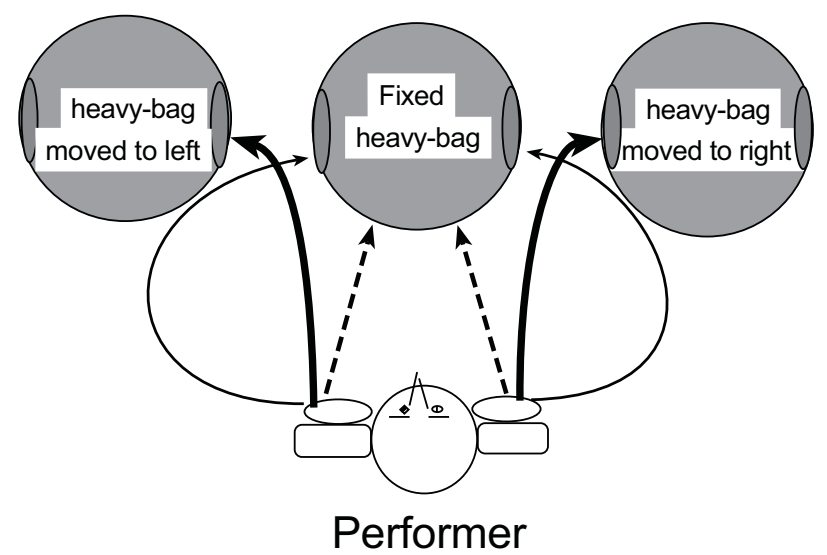

Fig. (4). For the scaled distances: $0.0 \leq \mathrm{D} \leq 0.4$, the heavybag was moved stochastically on left and right crossing the shoulder width of the performers. This relaxation of constraints led to invention of a novel punch (the bold arrow). (with kind permission from Nonlinear Dynamics, Psychology and Life Sciences).

action of ipsilateral, i.e. same-sided, coupling of hooks with lateral heavy-bag markers. Moreover, one of these performers still maintained the front-fist - target collision surface but compensated that with a dorsal (i.e. backward) flexion in the wrist. The other one discovered the proper back-fist karate punch. The other two performers were trapped within the same-sided interaction with the marked lateral surfaces on the heavy-bag. These performers needed some verbal support (within the observation time) in the form of instructional constraint, i.e. the question: How else would you strike the left-right side marked area? Note that this intervention was not an explicit instruction of how to perform the punch, but just perturbed the more stable performer-target coupling and enabled the back-fist punch to be discovered. In other words what these performers discovered was a new affordance (opportunity for action). This is a typical case of crossfertilization of two socio-cultural task-solution landscapes within martial arts. The emergence of Jeet Kune Do martial art is a potent example of such process. This case of blending task-goal solutions forms a specific type of creative behavior [52]. Note that this type of originality arises through a task reconfiguration, which is also a specific strategy of creative behavior: striking a heavy bag is not just boxing task.

For the first two performers the enhanced environmental variability was sufficient to discover the novel action and they were able to use the short time window of the heavybag maximal elongation to discover the new affordance. Obviously, the environmental variability relaxed the task constraints within the performer-environment system and formed a new attractive state in the dynamical landscape of these performers. The other two performers needed an additional verbal perturbation to intentionally direct the attention and explore other action possibilities. Nevertheless, in all four performers, the suppression of habitual actions released task constraints of classical boxing punches (e.g. front-fist contact with the target, zero wrist angle) and led to an innovation within the performer's dynamic landscape. In other words, the stringent constraints in the latter two performers, i.e. stopping the habitual action, also led to releasing of other correlated task constraints and formation of a novel interaction with environment.

From these results it follows that the releasing of task, as well as other classes of constraints, is a condition that facilitates the exploratory breadth $\mathrm{Q}$, the fluency and flexibility as well as originality within the domain of sports problem solving (see Fig. 5). Perhaps, anything that leads to instability of the habitual action may lead to the invention of a new action. This observation demonstrates how certain sets of constraints enable larger exploratory breadth, fluency and flexibility of athlete-environment dynamics and how originality (atypical-

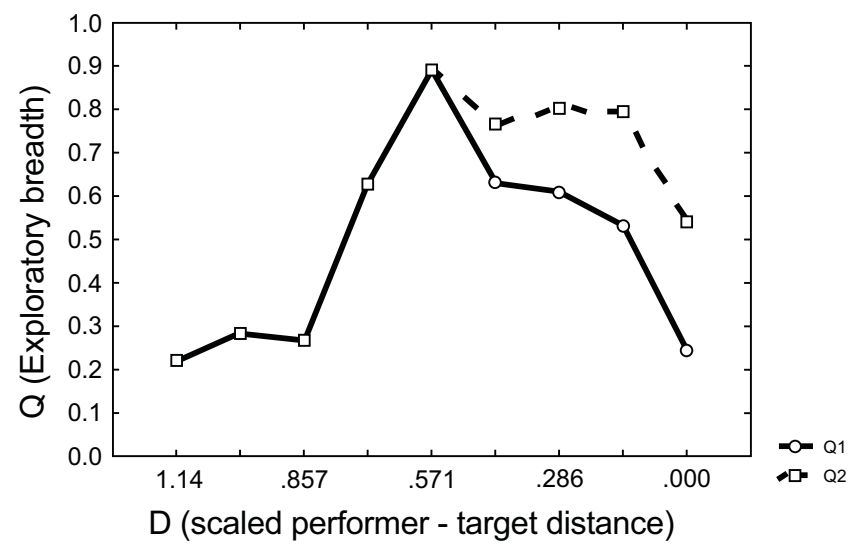

Fig. (5). The exploratory breadth of the performer - target system was larger for the stochastically moved heavy-bag (dashed line) than for the fixed heavy-bag (bold line) constraints. Larger degree of fluency, flexibility and atypicality was found under released task constraints. (with kind permission from Nonlinear Dynamics, Psychology and Life Sciences). 
ity) may be largely enhanced by small interventions in task constraints (see Fig. 5). Such atypical actions may form the basis of the cross-fertilization process between different sports disciplines which may lead to the emergence of new sport disciplines and form a Big $\mathrm{C}$ creative behavior.

It is important to note that the emergence of short term action possibilities, i.e. affordances, together with the reorganization of action degrees of freedom were hallmarks of the creative discovery behavior of performers. This may be an important property of creative actions in individual and collective sports either of small c creativity or Pro C creativity in Beghetto-Kaufman's [45] classification. This property of fluency and flexibility may be figuratively termed a "connected doors property" in a sense that creative performerenvironment systems afford a quick opening of the door, i.e. action possibility, at the moment when the other door (affordance) was being shut. This property is a consequence of the metastable dynamical regime within such systems, which do not form one or few highly stable states from which it is hard to escape, but dwell in the weakly stable or weakly unstable perception-action regions [27] which enables quick switches between actions. Such property is instrumental in creative, i.e. flexible, fluent and original, behaviors which emerge also within the rules of extant disciplines.

\section{CREATIVE BEHAVIOR IN TEAM SPORTS}

Creativity in team sports is sustained by the nonlinear interactions among players. As in any other social system the way that each player interacts with others in the neighborhood of play influences the behaviors of players within the same team and this is a requisite to disturb the actions of opponents [37].

From an attacker's perspective the decisions of the ball carrier and support players are based on the perceptions that they have created of the defenders' relative positioning, running line trajectories, and proximity to each other. On the other hand, the decision making of defenders depends on the perception that they have of the ball carrier's actions as well as the behavious of the support players. These variables include interpersonal distances, the speed and running line trajectories that contain important information concerning the attackers' ability to perform different actions. These variables contain information that are perceived by the players and specify the action possibilities of each opponent or teammate $[53,54]$. This is where creativity emerges, with the need for attackers to perform deceptive actions that creates the impression of multiple different possibilities for action. These deceptive actions can also be characterized by intrateam coordination where attackers perform a set of previous established movements that are intended to open a spacetime window against a stable opposing team. This is when creativity is needed again and players need to reorganize, avoiding defenders. This reorganization process is grounded on situational information concerning defenders' relative positions, number, speed and distance to goal [26,55-57]. These sources function as task constraints that attackers use to avoid defenders. The reorganization of attackers is grounded on situational information that emerges due to opponent players' nonlinear interactions and is selforganized.
Typically attacker-defender interactions are characterized with many subtle fluctuations in the attacker-defender balance but also with few abrupt changes in the attackerdefender structural organization, meaning that suddenly the attackers gain an advantage and are in a crucial position to score.

\section{The Need of Playing Within Critical Regions of Interper- sonal Distance}

Attackers' interactions aim to actively explore space-time windows that emerge due to defenders' displacements. On the other hand, defenders' displacements aim to cover the possible paths to goal, which demands high levels of interpersonal coordination among the players in defense. However space-time windows will only emerge if the attackers' movements are powerful enough to disturb the defenders' interpersonal coordination, and to do that, attackers' actions must be performed within short distances of attackerdefender interpersonal distance [32].

Thus sudden changes in the attacker-defender structural organization can only happen when the attacker-defender systems moved towards regions of very short interpersonal distances where the contextual dependency among players emerge characterizing the performance region as critical. Within these critical regions the player's contextual dependency move the system from equally poised options to a single one, that emerge under the influence of task and environmental constraints. In other words within these critical regions creativity occurs as ongoing trial solutions emerge and are annihilated, until a sudden change occurs where a single solution (i.e., creativity) emerges [33]. The players' contextual dependency creates local information that originate a specific moment in time and space where a gap on the defense emerge and the attackers use it to take advantage getting close to the goal or even score, this is why we support the notion that creativity in team sports is based upon a self-organization mechanism that only occur within critical regions.

\section{CONCLUSION}

In summary, we showed how the general principles of CDS may lead to experiments in which one can study the idiosyncratic interactions of task, personal and environmental constraints within the performer-environment system leading to action discovery and innovative behavior. We showed how the hierarchical landscape of exploration emerges and gets soft-assembled under different sets of constraints and how fluency, flexibility and originality may be defined on more levels.

Further, two strategies of creativity facilitation and exploratory breadth were obtained as important: Direct relaxation of key constraints, i.e. situating the performer under such set of constraints that afford larger exploration of taskgoal space; and indirect releasing of constraints (suppressing habitual action). The combination of task goal constraint and the suppressed habitual action (by an opponent action or by the athlete her/himself) releases other constraints configurations from which a novel action form emerges. This may be observed at different time scales [17]: from immediate situational movement adaptation (e.g., a basketball player 
actions) to many months and years of development of the invented technique.

The creative athlete-environment interaction is based on an athlete's self-experimentation with constraint configurations. For any set of interacting constraints a movement emerges spontaneously. It follows that a novel athleteenvironmental interaction is predicated on a novel constellation of constraints that athlete discovers by experimentation.

For understanding the creativity in team sports it is very important to realize that under the confluence of constraints such as, the inter-individual distance, relative speed of athletes a special critical region is formed characterized by high level of possible action solutions each of which is idiosyncratic and formed by specific set of interacting constraints. Such, idiosyncratic emergent solutions are very often created by the context formed by collective actions of the whole team.

\section{CONFLICT OF INTEREST}

The author(s) confirm that this article content has no conflicts of interest.

\section{ACKNOWLEDGEMENT}

Declared none.

\section{REFERENCES}

[1] Guilford JP. The structure of intellect. Psychol Bull 1956; 53 (4): 267-93.

[2] Esteves PT, de Oliveira RF, Araújo D. Posture-related affordances guide attacks in basketball. Psychol Sport Exerc 2011; 12(6): 63944.

[3] Torrance EP. The torrance tests of creative thinking-normstechnical manual research edition-verbal tests, forms A and Bfigural tests, forms A and B. Princeton, NJ: Personnel Press 1966.

[4] Lee J. Dick Fosbury: Former Olympic high jumper. 2007. Available at: http://speedendurance.com/2007/06/15/dick-fosburyformer olympic-high-jumper/. [Accessed Nov 19 ${ }^{\text {th }} 2011$ ].

[5] Amabile TM. Attributions of creativity: what are the consequences? Creat Res J 1995; 8: 423-6.

[6] Ford CM. A theory of individual creative action in multiple social domains. Acad Manage Rev 1996; 21(4):1112-42.

[7] Kasof J. Explaining creativity: the attributional perspective. Creat Res J 1995; 8(4): 311-66.

[8] Mumford MD, Simonton DK. Creativity in the workplace: people, problems, and structures. J Creat Behav 1997; 31(1):1-6.

[9] Runco MA, Charles R. Judgments of originality and appropiateness as predictors of creativity. Pers Individ Dif 1992; 15: 537-46.

[10] Runco MA. Creativity. Annu Rev Psychol 2004; 55: 657-87.

[11] Sternberg RJ, Lubart TI. Investing in creativity. Am Psychol 1996; 51(7): 677-88

[12] Edelman GM, Gally JA. Degeneracy and complexity in biological systems. Proc Natl Acad Sci U S A 2001; 98(24):13763-8.

[13] Schmidt RA. Motor schema theory after 27 years: reflections and implications for a new theory. Res Q Exerc Sport 2003;74(4): 36675 .

[14] Ratcliff R, Van Zandt T, McKoon G. Connectionist and diffusion models of reaction time. Psychol Rev 1999; 106(2): 261-300.

[15] Stephen DG, Dixon JA. The self-organization of insight: entropy and power laws in problem solving. J Problem Solving 2008; 2(1): 72-101.

[16] Hristovski R. On the dynamics of biomotor actions as state changes in a human biomotor field (In Serbian). Fizicka Kultura 1989; 43: 59-63.

[17] Newell KM, Liu YT, Mayer-Kress G. Time scales in motor learning and development. Psychol Rev 2001; 108(1): 57-82.
[18] Wiggins GA, Ed. Towards a more precise characterisation of creativity in AI. Proceedings of the ICCBR 2001 Workshop on Creative Systems. Vancouver: British Columbia 2001.

[19] Newell KM. Constraints on the development of coordination. In: Wade MG, Whiting HTA, Eds. Motor development in children. Aspects of coordination and control. Dordrecht, Netherlands: Martinus Nijhoff 1986; pp. 341-60.

[20] Davids K, Shuttleworth R, Button C, Zealand N. Acquiring skill in sport : a constraints-led perspective. Int J Comput Sci Sport 2003; 2(2):31-9.

[21] Araújo D, Davids K, Bennett S, Button C, Chapman G. Emergence of sport skills under constraints. In: Williams AM, Hodges NJ, Eds. Skill acquisition in sport: Research, theory and practice. London: Routledge 2004; pp. 409-33.

[22] Chow JY, Davids K, Button C, Shuttleworth R, Renshaw I, Araujo D. Nonlinear pedagogy: a constraints-led framework for understanding emergence of game play and movement skills. Nonlinear Dynamics Psychol Life Sci 2006; 10(1):71-103.

[23] Davids K, Renshaw I, Glazier P. Movement models from sports reveal fundamental insights into coordination processes. Exerc Sport Sci Rev 2005; 33(1): 36-42.

[24] Davids K, Button C, Bennett S. Dynamics of skill acquisition. A constraints-led approach.Champaign: Human Kinetics 2008.

[25] Araujo D, Davids K, Hristovski R. The ecological dynamics of decision making in sport. Psychol Sport Exerc 2006; 7(6): 653-76.

[26] Araújo D, Davids K, Chow JY, Passos P. The development of decision making skill in sport: an ecological dynamics perspective. In: Araújo D, Ripoll H, Raab M, Eds. Perspectives on cognition and action in sport. New York: Nova Science Publishers 2009; pp.157-70.

[27] Chow JY, Davids K, Hristovski R, Araújo D, Passos P. Nonlinear pedagogy: learning design for self-organizing neurobiological systems. New Ideas Psychol 2011; 29(2):189-200.

[28] Davids K, Araujo D. The concept of 'Organismic Asymmetry' in sport science. J Sci Med Sport 2010; 13(6): 633-40.

[29] Araújo D, Davids K. What exactly is acquired during skill acquisition. J Conscious Stud 2011;18(3-4):23.

[30] Araújo D, Davids K. Talent development: from possessing gifts to functional environmental interaccions. Talent Dev Excell 2011; 1: 25.

[31] Hristovski R, Davids K, Araujo D. Affordance-controlled bifurcations of action patterns in martial arts. Nonlinear Dynamics Psychol Life Sci 2006; 10(4): 409-44.

[32] Passos P, Araujo D, Davids K, Gouveia L, Milho J, Serpa S. Information-governing dynamics of attacker-defender interactions in youth rugby union. J Sports Sci 2008; 26(13):1421-9.

[33] Passos P, Araujo D, Davids K, et al. Interpersonal pattern dynamics and adaptive behavior in multiagent neurobiological systems: conceptual model and data. J Mot Behav 2009; 41(5):445-59.

[34] Hristovski R, Davids K, Araújo D. Information for regulating action in sport: metastability and emergence of tactical solutions under ecological constraints. In: Araújo D, Ripoll H, Raab M, Eds. Perspectives on cognition and action in sport. Hauppauge, NY: Nova Science Publishers 2009; pp. 43-57.

[35] Hristovski R, Davids K, Araujo D, Passos P. Constraints-induced emergence of functional novelty in complex neurobiological systems: a basis for creativity in sport. Nonlinear Dynamics Psychol Life Sci 2011; 15(2):175-206.

[36] Turvey MT. Affordances and prospective control: an outline of the ontology. Ecol Psychol 1992; 4(3):173-87.

[37] Fajen BR, Riley MA, Turvey MT. Information, affordances, and the control of action in sport. Int J Sports Psychol 2009; 40(1):79107.

[38] Fajen BR, Turvey MT. Perception, categories, and possibilities for action. Adapt Behav 2003; 11(4):276-8.

[39] Gibson JJ. The ecological approach to visual perception.. Hillsdale, New Jersey: Lawrence Erlbaum Associates 1979.

[40] Beek PJ, Dessing JC, Peper CE, Bullock D. Modelling the control of interceptive actions. Philos Trans R Soc Lond B Biol Sci 2003; 358(1437):1511-23.

[41] Turvey MT, Shaw RE. Toward an ecological physics and a physical psychology. In: Solso RL, Massaro DW, Eds. The Science of the Mind: 2001 and Beyond. New York: Oxford University Press 1995; pp.144-69.

[42] Davids K, Button C, Araújo D, Renshaw I, Hristovski R. Movement models from sports provide representative task constraints for 
studying adaptive behavior in human motor systems. Adapt Behav 2006; 14:73-95.

[43] Hristovski R, Davids K, Araújo D, Button C. How boxers decide to punch a target: emergent behaviour in nonlinear dynamical movement systems. J Sports Sci Med 2006; 5(CSSI-1): 60-73.

[44] Kelso JAS, Engstrøm DA. The complementary nature. Cambridge, MA, US: The MIT Press 2006.

[45] Beghetto RA, Kaufman JC. Toward a broader conception of creativity: A case for "mini-c" creativity. Psychol Aesthet Creat Arts 2007; 1(2): 73-9.

[46] Liu YT, Mayer-Kress G, Newell KM. Qualitative and quantitative change in the dynamics of motor learning. J Exp Psychol Hum Percept Perform 2006; 32(2): 380-93.

[47] Simonton DK. Creativity as blind variation and selective retention: is creative process Darwinian? Psychol Inq 1999; 10(4): 309-28.

[48] Memmert D, Furley P. "I spy with my little eye!": breadth of attention, inattentional blindness, and tactical decision making in team sports. J Sport Exerc Psychol 2007; 29(3): 365-81.

[49] Hristovski R, Balague N. Fatigue-induced spontaneous termination point-nonequilibrium phase transitions and critical behavior in quasi-isometric exertion. Hum Mov Sci 2010; 29(4): 483-93.
[50] Sporns O. Complex Neural Dynamics. In: Jirsa V, Kelso JAS, Eds. Coordination dynamics: Issues and trends. Berlin, Heidelberg: Springer-Verlag 2004.

[51] Haken H. Synergetics: an introduction: nonequilibrium phase transitions and self-organization in physics, chemistry, and biology. $3^{\text {rd }}$ ed. Berlin: Springer 1983.

[52] Sternberg R. Wisdom, inteligence and creativity synthesized. Cambridge: Cambridge University Press 2003.

[53] Gunns RE, Johnston L, Hudson SM. Victim selection and kinematics: a point-light investigation of vulnerability to attack. J Nonverbal Behav 2002; 26(3):129-58

[54] Weast JA, Shockley K, Riley MA. The influence of athletic experience and kinematic information on skill-relevant affordance perception. Q J Exp Psychol (Hove) 2011; 64(4): 689-706.

[55] Travassos B, Araujo D, Vilar L, McGarry T. Interpersonal coordination and ball dynamics in futsal (indoor football). Hum Mov Sci 2011; 30(6): 1245-59.

[56] Cordovil R, Araujo D, Davids K, et al. The influence of instructions and body-scaling as constraints on decision-making processes in team sports. Eur J Sport Sci 2009; 9(3): 169-79.

[57] Passos P, Milho J, Fonseca S, Borges J, Araujo D, Davids K. Interpersonal distance regulates functional grouping tendencies of agents in team sports. J Mot Behav 2011; 43(2): 155-63.

(C) Hristovski et al.; Licensee Bentham Open.

This is an open access article licensed under the terms of the Creative Commons Attribution Non-Commercial License (http://creativecommons.org/licenses/ by-nc/3.0/) which permits unrestricted, non-commercial use, distribution and reproduction in any medium, provided the work is properly cited. 